\title{
Association between five types of Tumor Necrosis Factor-a gene polymorphism and hepatocellular carcinoma risk: a meta- analysis
}

Citrawati Dyah Kencono Wungu ${ }^{1,2^{*}}$ (D) Fis Citra Ariyanto ${ }^{3,4}$ (D), Gwenny Ichsan Prabowo ${ }^{1}$, Soetjipto ${ }^{1,2}$ and Retno Handajani ${ }^{1,2}$

\begin{abstract}
Background: Research focusing on the relationship between five types of tumor necrosis factor-alpha (TNF-a) SNPS and the risk of hepatocellular carcinoma (HCC) were still controversial. Hereby, we performed a meta-analysis to determine the association between TNF-a promoter SNPs: -1031 T/C, -863 C/A, -857 C/T, $-308 \mathrm{G} / \mathrm{A}$, and - $238 \mathrm{G} /$ A with HCC risk.

Methods: We interrogated articles from journal database: PubMed, Pro-Quest, EBSCO, Science Direct, and Springer to determine the relationship between five types of SNPs in TNF-a gene with HCC risk. RevMan 5.3 software was used for analysis in fixed/random effect models.

Results: This meta-analysis included 23 potential articles from 2004 to 2018 with 3237 HCC cases and 4843 controls. We found that SNP - $863 \mathrm{C} / \mathrm{A}$ were associated with a significantly increased HCC risk (A vs C, OR $=1.31$, $95 \% \mathrm{Cl}=1.03-1.67)$. Similar results were obtained in $-857 \mathrm{C} / \mathrm{T}(\mathrm{TT} / \mathrm{CT}$ vs $\mathrm{CC}, \mathrm{OR}=1.31,95 \% \mathrm{Cl}=1.06-1.62),-308 \mathrm{G} /$ $\mathrm{A}$ ( $\mathrm{AA}$ vs $\mathrm{GG}, \mathrm{OR}=3.14,95 \% \mathrm{Cl}=2.06-4.79$ ), and $-238 \mathrm{G} / \mathrm{A}$ (AA vs $\mathrm{GG}, \mathrm{OR}=3.87,95 \% \mathrm{Cl}=1.32-11.34$ ). While no associations were observed between SNP TNF- $a-1031$ T/C and HCC risk.
\end{abstract}

Conclusions: The present meta-analysis showed that TNFa SNPs $-863 C / A,-857 \mathrm{C} / \mathrm{T},-308 \mathrm{G} / \mathrm{A}$, and $-238 \mathrm{G} / \mathrm{A}$ were associated with the risk of HCC.

Keywords: Meta-analysis, Tumor necrosis factor-a, Single nucleotide polymorphism, Hepatocellular carcinoma

\section{Introduction}

Hepatocellular carcinoma (HCC) or liver cancer accounts for the cancer with the fourth highest mortality rate in the world. In 2018, there are estimated to be 841 , 080 of HCC new cases worldwide [1]. Patients with HCC usually have poor prognosis and high mortality

\footnotetext{
* Correspondence: citrawati.dyah@fk.unair.ac.id

'Department of Medical Biochemistry, Faculty of Medicine, Universitas Airlangga, Surabaya, Indonesia

${ }^{2}$ Institute of Tropical Disease, Universitas Airlangga, Surabaya, Indonesia Full list of author information is available at the end of the article
}

rates, even in developed countries [2]. Until now, the pathogenesis of HCC has not been fully understood, but it is known so far that it is influenced by hepatitis B and $\mathrm{C}$ virus infections and also influenced by environmental factors (smoking, alcohol, aflatoxin B1) [3]. It is known that there are differences in the risk of $\mathrm{HCC}$ within each person, in which case the host factor has an important role [4].

Tumor Necrosis Factor- $\alpha$ (TNF- $\alpha$ ) is an important inflammatory cytokine in the development of liver disease. This cytokine can cause hepatic injury, cirrhosis and

(c) The Author(s). 2020 Open Access This article is licensed under a Creative Commons Attribution 4.0 International License, which permits use, sharing, adaptation, distribution and reproduction in any medium or format, as long as you give appropriate credit to the original author(s) and the source, provide a link to the Creative Commons licence, and indicate if changes were made. The images or other third party material in this article are included in the article's Creative Commons licence, unless indicated otherwise in a credit line to the material. If material is not included in the article's Creative Commons licence and your intended use is not permitted by statutory regulation or exceeds the permitted use, you will need to obtain permission directly from the copyright holder. To view a copy of this licence, visit http://creativecommons.org/licenses/by/4.0/. The Creative Commons Public Domain Dedication waiver (http://creativecommons.org/publicdomain/zero/1.0/) applies to the data made available in this article, unless otherwise stated in a credit line to the data. 
eventually promote hepatocellular carcinoma $[5,6]$. Several previous studies have identified some Single Nucleotide Polymorphism (SNP) $s$ in TNF- $\alpha$ gene, especially in the promoter region. SNPs of TNF- $\alpha-1031 \mathrm{~T} / \mathrm{C}$ (rs1799964), $-863 \mathrm{C} / \mathrm{A} \quad(\mathrm{rs} 1800630), \quad-857 \mathrm{C} / \mathrm{T}$ (rs1799724), -308G/A (rs1800629), and -238G/A (rs361525) are SNPs in the TNF- $\alpha$ promoter site that have often been investigated regarding their association with $\mathrm{HCC}$ in several previous studies $[7,8]$. It was also said that those SNPs could affect TNF- $\alpha$ production at the transcription level $[9,10]$. This plays important role because cytotoxic $\mathrm{T}$ lymphocytes (CTLs) in the liver secrete TNF- $\alpha$ [11]. Increased TNF- $\alpha$ level due to those SNPs can cause persistent inflammatory condition in liver tissue which is the most important risk factor for HCC [7].

High production of TNF-is related to the increase of pro-inflammatory cytokine secretion, the activation of proto oncogenes and several genes associated with cell growth, invasion, and cancer cells metastasis [12, 13]. Excessive production of TNF $\alpha$ can also induce the generation of free radicals in the form of Reactive Oxygen Species which can cause further liver damage and genomic instability [14]. It is also said that high TNF- $\alpha$ expression is an independent predictor of poor survival in HCC patients [15].

The results of various previous studies regarding the relationship between TNF- $\alpha$ polymorphism and HCC across various ethnicities and populations show mixed results, related [16-18] or there is no relationship [19, 20]. Research on TNF- $\alpha$ gene SNPs in patients with Hepatitis B Virus (HBV) and Hepatitis C Virus (HCV) infection is specific for each ethnicity and shows different results in each population [21, 22]. As the results regarding the role of these five SNPs of TNF- $\alpha$ genes against $\mathrm{HCC}$ are still controversial, we conducted this meta-analysis to determine the relationship between those TNF- $\alpha$ gene polymorphisms and HCC.

\section{Methods}

\section{Database searching}

Our meta-analysis was reported based on the items outlined in the Preferred Reporting Items for Systematic Reviews and Meta-Analyses (PRISMA) statement to ensure adequate reporting of this meta-analysis of observational studies in epidemiology [23]. We conducted an electronic database searching to identify all previously published cohort or case-control studies investigating the association between five types of TNF- $\alpha$ gene SNPs ($1031 \mathrm{~T} / \mathrm{C},-863 \mathrm{C} / \mathrm{A},-857 \mathrm{C} / \mathrm{T},-308 \mathrm{G} / \mathrm{A}$, and $-238 \mathrm{G} / \mathrm{A}$ ) and risk of HCC. We searched data from PubMed, ProQuest, EBSCO, Science Direct, and Springer by using MeSH terms: "Tumor Necrosis Factor-alpha" or "TNFalpha" and "polymorphism" or "SNP" or "single nucleotide polymorphism" or "variant" and "hepatocellular carcinoma" or "HCC" or "liver cancer". The search was conducted in September 2019-January 2020. We also performed a manual search was also performed to obtain potential sources cited in other meta-analysis.

\section{Criteria for inclusion and exclusion}

All included studies should meet the following criteria: (1) investigate the association between any of the five SNPs $(-1031 \mathrm{~T} / \mathrm{C},-863 \mathrm{C} / \mathrm{A},-857 \mathrm{C} / \mathrm{T},-308 \mathrm{G} / \mathrm{A}$, and -238G/A) in TNF- $\alpha$ gene and risk of HCC; (2) have cohort or case-control study design; (3) risk of HCC was reported as RR (relative risk) and/or OR (odds ratio) with 95\% Confidence Interval (CI) or provide sufficient data to extract RR and/or OR with 95\% CI data; (4) include human subjects; (6) in English language. We excluded studies with the following criteria: (1) studies with design other than case-control or cohort; (2) duplicated studies; (3) studies with unmeasurable population and not qualified data; (4) using non-English language; (5) Studies in which the full text or main data could not be obtained.

\section{Study selection}

Two investigators independently performed the electronic search and retrieved the articles that matched with our searched terms. Any disagreement was settled by discussion and consensus with all the authors. Final decision was merely based on the agreements of all authors.

\section{Data extraction}

A standardized reporting form was used to extract the data from each article which included the first author's name, year of publication, population country, TNF- $\alpha$ SNP type, study design, etiology of HCC, SNP genotyping method, controls, and frequencies of SNP. HardyWeinberg equilibrium test was performed and its significance of the control groups was calculated when the original information was not provided.

\section{Quality assessment}

Newcastle - Ottawa quality assessment scale (NOQS) was used for measuring the quality of the included studies. This scale was designed through collaboration between the Universities of Newcastle, Australia and Ottawa, Canada. The purpose of this scale was to assess the quality of observational studies for producing good meta-analysis. The studies were qualified as high quality (9 stars), medium quality (7-8 stars), and low quality (less than 7 stars) [24]. 


\section{Data analysis}

Analysis were conducted using Review Manager 5.3 software (The Cochrane Collaboration, UK). HardyWeinberg Equilibrium was examined by Chi Square test when the original information was not provided. We evaluate 5 genetic models (allele, dominant, recessive, codominant major vs minor homozygote, and codominant heterozygote vs major homozygote) for each SNP type separately. Major alleles for each SNP were: -1031TT, -863CC, -857CC, -308GG, and -238GG, while minor alleles for each SNP were: -1031CC, -863AA, -857TT, -308AA, and -238AA. Heterogeneity assumption was assessed with Cochrane $Q$ statistic and $\mathrm{I}_{2}$ statistic. The pool estimated ORs was calculated with either fixed or random effects model assumptions. If $\mathrm{Q}$ test showed significant result $(p<0.05)$, we used a random effects model. Otherwise, if $\mathrm{Q}$ test showed insignificant result $(p>0.05)$, we used a fixed effect model. We also calculated the $95 \%$ confidence interval (CI) of pool estimated OR. Inverted funnel plots were conducted to find any presence of publication bias.

\section{Results}

\section{Study selection}

According to the PRISMA flow diagram (Fig. 1), we initially obtained 29,182 studies through primary database searching and 12 through manual searching. After screening the titles/abstracts we selected 374 potentially relevant articles. Among them, 260 were excluded due to non-English language (19 studies) and unsuitable study designs (review papers/case reports/ cross sectional/meta-analysis/experimental; 241 studies). Then, 114 full text studies were checked for their eligibility. Some studies were excluded due to duplicates or irrelevant study design/insufficient information/unqualified articles until we finally obtained 23 included studies.

Eventually, as much as 23 potential articles were included which recruited 9792 participants and consisted of $3237 \mathrm{HCC}$ cases and 4843 controls (Table 1). The papers were published 2004 to 2018 . Each study has sample size ranged from 45 to 1624 participants. Of all included studies in this meta-analysis, most studies came from Asia, in which 7 studies were from Mainland China [7, 25-30], 4 from Taiwan [16, 31-33], 2 from India [19, 34], 2 from Korea [8, 35], 1 from Japan [36], 1 from Thai [37], and 1 from Turkey [38]. There were also some studies with non-Asian countries, including 2 from Egypt [39, 40], 1 from Brazil [41], 1 from Italy [42], and 1 from Tunisia [43]. From the 23 included studies, the aetiology of HCC was mostly caused by HBV (12 studies), followed by mixed cause (8 studies), HCV (2 studies), and alcohol/smoking (1 study). Some studies observed 1 locus, and others observed more than 1 loci. Studies observing polymorphism at -308 was the most frequent (19 studies), while SNP - 1031 was the less frequently observed (5 studies). Allele frequencies of SNPs in each population is showed on Table 2.

\section{Association between SNP TNF-a - 1031 and HCC risk}

Only five studies regarding the association between SNP TNF- $\alpha-1031$ and HCC risk with 825 cases and 1518 controls were available. The number of cases for $\mathrm{CC}$ and TC genotypes was reported together in the studies by Niro et al. [42] and Jin et al. [35] which could only be used for dominant-model analysis (CC/TC vs TT; Table 3). As the heterogeneity among studies for all models $\left(\mathrm{I}_{2}\right)$ was less than $60 \%$ and $p$-value for the heterogeneity was more than 0.05 , fixed-effects models were applied. However, we did not obtain any significant association between SNP TNF- $\alpha-1031$ and HCC risk in all model analyses. For the estimation of publication bias, we found no visual asymmetry in Funnel Plot analysis.

\section{Association between SNP TNF-a - 863 and HCC risk}

We included eight studies with 1642 cases and 2746 controls to determine the association between SNP TNF- $\alpha-863$ and HCC risk. The numbers of cases for $\mathrm{CA}$ and AA genotypes were reported together in the study by Niro et al. [42], thus it could only be used for dominant-model analysis (AA/CA vs CC; Table 3). In studies with heterogeneity among studies $\left(\mathrm{I}_{2}\right)$ more than $60 \%$ and $p$-value for the heterogeneity was less than 0.05 , we applied random-effects models. We found significant association between the allele model of A versus $\mathrm{C}$ of TNF- $\alpha \mathrm{C} / \mathrm{A}$ SNP with the risk of $\mathrm{HCC}(\mathrm{OR}=1.31$, 95\% CI $=1.03-1.67, p=0.03)$. The dominant-model analysis (CA/AA vs CC) also showed significant association between SNP TNF- $\alpha 863 \mathrm{C} / \mathrm{A}$ and HCC risk (OR = 1.19, 95\% CI $=1.03-1.36, p=0.02$; Fig. 2). As heterogeneity was found in the statistical analyses, we did sensitivity analyses to evaluate the sources of heterogeneity. We found that heterogeneity between studies was mainly caused by the study by Kummee et al. [37], as after this study was excluded, no significant heterogeneity was found.

\section{Association between SNP TNF-a - 857 and HCC risk}

We only obtained five studies about the association between SNP TNF- $\alpha-857$ and HCC risk with 716 cases and 1005 controls. The number of cases for TT and CT genotypes was reported together in the studies by Jin et al. [35] which could only be used for dominant-model analysis (TT/CT vs CC; Table 3). As the heterogeneity among studies for all models $\left(\mathrm{I}_{2}\right)$ was less than $60 \%$ and $p$-value for the heterogeneity was more than 0.05 , fixedeffects models were applied. A significant association between SNP TNF- $\alpha-857 \mathrm{C} / \mathrm{T}$ and $\mathrm{HCC}$ risk was found in dominant-model analyses $(\mathrm{OR}=1.31,95 \% \mathrm{CI}=1.06-$ 


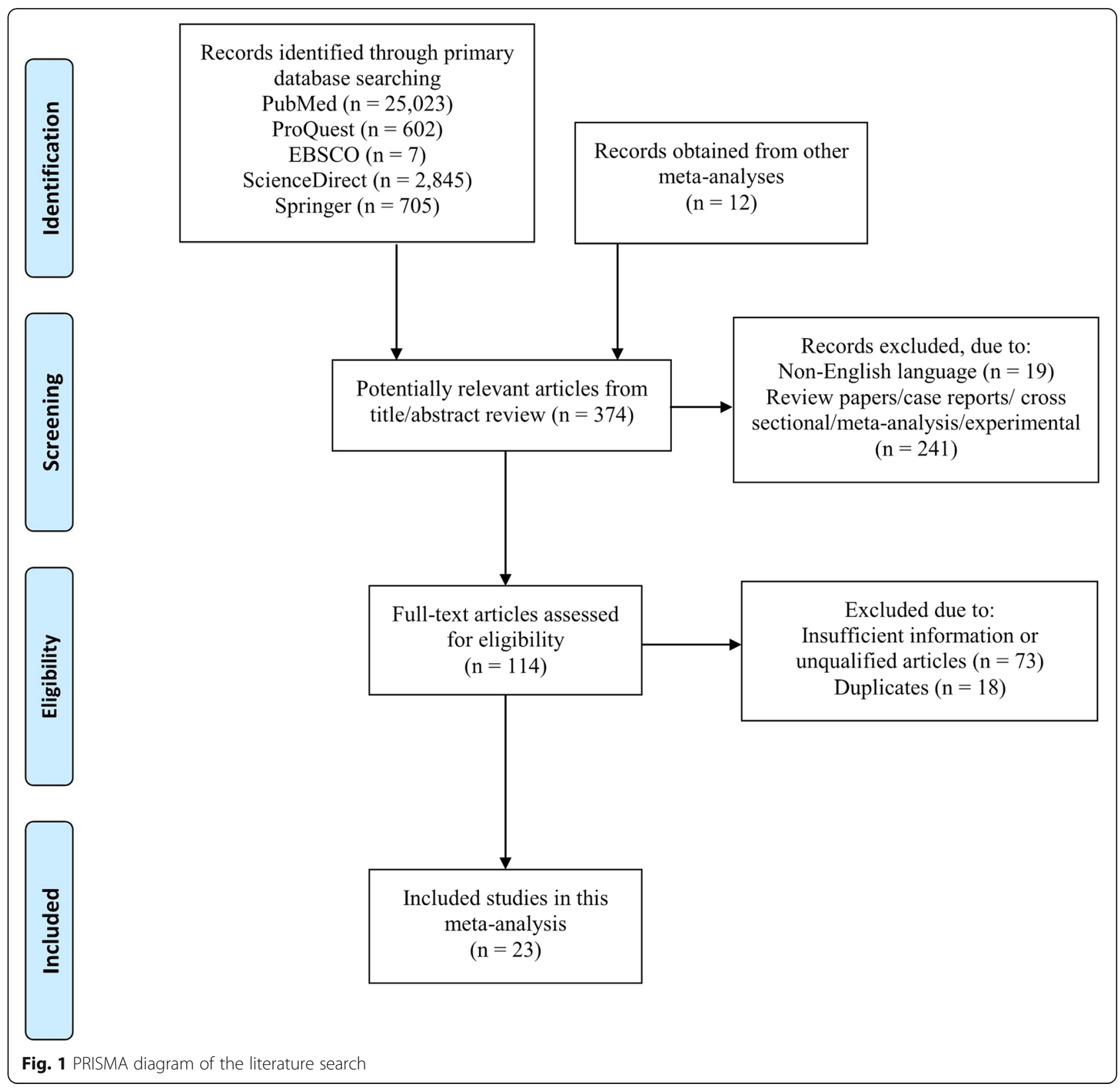

1.62, $p=0.01$; Fig. 3). We did not observe any visual asymmetry in Funnel Plot analysis regarding the publication bias.

\section{Association between SNP TNF-a - 308 and HCC risk}

Interestingly, we found most included studies analyzing the association between SNP TNF- $\alpha-308 \mathrm{G} / \mathrm{A}$ and $\mathrm{HCC}$ risk. The number of cases for AA and GA genotypes was reported together in the studies by Niro et al. [42] and Jin et al. [35] which could only be used for dominant-model analysis (AA/GA vs GG; Table 3). In studies with heterogeneity among studies $\left(\mathrm{I}_{2}\right)$ more than $60 \%$ and $p$-value for the heterogeneity was less than 0.05 , random-effects models were applied. All five genetic models showed significant association with risk of $\mathrm{HCC}$ with OR for allele model $=1.98,95 \% \mathrm{CI}=1.62$ 2.42, $p<0.001$; OR for dominant model $=1.95,95 \% \mathrm{CI}=$ $1.53-2.49, p<0.001$; OR for recessive model $=2.52$, $95 \%$ $\mathrm{CI}=1.69-3.76, p<0.001$; OR for codominant major vs minor homozygote model $=3.14,95 \% \mathrm{CI}=2.06-4.79$, $p<0.001$; and OR for codominant heterozygote vs major homozygote model $=2.07,95 \% \mathrm{CI}=1.60-2.68, p<0.001$; Fig. 4). As heterogeneity was found in the statistical analyses, we did sensitivity analyses to evaluate the sources of heterogeneity. We found that heterogeneity between studies was mainly caused by the studies by $\mathrm{Ho}$ et al. [31] and Akkiz et al. [38], as after these studies were excluded, no significant heterogeneity was found. 
Table 1 The basic characteristics of the included studies

\begin{tabular}{|c|c|c|c|c|c|c|c|c|c|c|c|}
\hline \multirow[t]{2}{*}{ No } & \multirow{2}{*}{$\begin{array}{l}\text { First } \\
\text { author }\end{array}$} & \multirow[t]{2}{*}{ Year } & \multirow[t]{2}{*}{ TNF-a SNPs } & \multirow{2}{*}{$\begin{array}{l}\text { Study } \\
\text { design }\end{array}$} & \multicolumn{2}{|c|}{ Sample size } & \multirow[t]{2}{*}{ Population } & \multirow{2}{*}{$\begin{array}{l}\text { SNP } \\
\text { genotyping } \\
\text { method }\end{array}$} & \multirow{2}{*}{$\begin{array}{l}\text { HCC } \\
\text { etiology }\end{array}$} & \multirow[t]{2}{*}{ Control } & \multirow[t]{2}{*}{ NOQS } \\
\hline & & & & & $\begin{array}{l}\text { Case } \\
(n= \\
3237)\end{array}$ & $\begin{array}{l}\text { Control } \\
(n= \\
4843)\end{array}$ & & & & & \\
\hline 1 & Heneghan & 2004 & $-308 \mathrm{G} / \mathrm{A},-238 \mathrm{G} / \mathrm{A}$ & Case control & 98 & 75 & China & PCR-RFLP & Mixed & $\begin{array}{l}\text { Healthy } \\
\text { subjects }\end{array}$ & 8 \\
\hline 2 & $\mathrm{Ho}$ & 2004 & $-308 \mathrm{G} / \mathrm{A}$ & Case control & 74 & 289 & Taiwan & PCR-RFLP & Mixed & $\begin{array}{l}\text { Healthy } \\
\text { subjects }\end{array}$ & 8 \\
\hline 3 & Migita & 2005 & $-308 \mathrm{G} / \mathrm{A}$ & Case control & 48 & 188 & Japan & PCR-SSP & HBV & $\begin{array}{l}\text { HBV } \\
\text { without } \\
\text { HCC }\end{array}$ & 7 \\
\hline 4 & Niro & 2005 & $\begin{array}{l}-1031 \mathrm{~T} / \mathrm{C},-863 \mathrm{C} / \mathrm{A} \\
-308 \mathrm{G} / \mathrm{A},-238 \mathrm{G} / \mathrm{A}\end{array}$ & Case control & 30 & 96 & Italy & Sequencing & HBV & $S R$ & 7 \\
\hline 5 & Jeng & 2007 & $-308 \mathrm{G} / \mathrm{A}$ & Case control & 108 & 108 & Taiwan & Sequencing & Mixed & $\begin{array}{l}\text { Healthy } \\
\text { subjects }\end{array}$ & 9 \\
\hline 6 & Kummee & 2007 & $\begin{array}{l}-863 \mathrm{C} / \mathrm{A},-308 \mathrm{G} / \mathrm{A} \\
-238 \mathrm{G} / \mathrm{A}\end{array}$ & Case control & 50 & 150 & Thailand & PCR-RFLP & HBV & $\begin{array}{l}\text { Healthy } \\
\text { subjects }\end{array}$ & 7 \\
\hline 7 & Akkiz & 2009 & $-308 \mathrm{G} / \mathrm{A}$ & Case control & 110 & 110 & Turkey & PCR-RFLP & Mixed & $\begin{array}{l}\text { Healthy } \\
\text { subjects }\end{array}$ & 9 \\
\hline 8 & Wang & 2010 & $-308 \mathrm{G} / \mathrm{A},-238 \mathrm{G} / \mathrm{A}$ & Case control & 230 & 158 & China & Sequencing & HBV & SR & 8 \\
\hline 9 & Chen & 2011 & $\begin{array}{l}-1031 \mathrm{~T} / \mathrm{C},-863 \mathrm{C} / \mathrm{A} \\
-857 \mathrm{C} / \mathrm{T},-238 \mathrm{G} / \mathrm{A}\end{array}$ & Case control & 126 & 126 & China & Sequencing & HBV & $\begin{array}{l}\text { Healthy } \\
\text { subjects }\end{array}$ & 9 \\
\hline 10 & Shi & 2011 & $-308 \mathrm{G} / \mathrm{A}$ & Case control & 88 & 88 & China & PCR-RFLP & HBV & $\begin{array}{l}\text { Healthy } \\
\text { subjects }\end{array}$ & 9 \\
\hline 11 & Qiu & 2012 & $-863 \mathrm{C} / \mathrm{A},-857 \mathrm{C} / \mathrm{T}$ & Case control & 195 & 189 & China & PCR-RFLP & HBV & $\mathrm{SR}$ & 7 \\
\hline 12 & Radwan & 2012 & $-308 \mathrm{G} / \mathrm{A}$ & Case control & 128 & 160 & Egypt & PCR-RFLP & $\mathrm{HCV}$ & $\begin{array}{l}\text { Healthy } \\
\text { subjects }\end{array}$ & 8 \\
\hline 13 & Shi & 2012 & $-308 \mathrm{G} / \mathrm{A}$ & Case control & 73 & 116 & China & PCR-RFLP & Mixed & $\begin{array}{l}\text { Healthy } \\
\text { subjects }\end{array}$ & 8 \\
\hline 14 & Yang & 2012 & $-863 \mathrm{C} / \mathrm{A}$ & Case control & 772 & 852 & China & RT-PCR & Mixed & $\begin{array}{l}\text { Healthy } \\
\text { subjects }\end{array}$ & 7 \\
\hline 15 & Teixeira & 2013 & $-308 \mathrm{G} / \mathrm{A},-238 \mathrm{G} / \mathrm{A}$ & Case control & 111 & 202 & Brazil & PCR-SSP & Mixed & $\begin{array}{l}\text { Healthy } \\
\text { subjects }\end{array}$ & 5 \\
\hline 16 & Panigrahi & 2014 & $\begin{array}{l}-863 \mathrm{C} / \mathrm{A},-857 \mathrm{C} / \mathrm{T} \\
-238 \mathrm{G} / \mathrm{A}\end{array}$ & Case control & 14 & 85 & India & PCR-RFLP & HBV & $\begin{array}{l}\text { Healthy } \\
\text { subjects }\end{array}$ & 6 \\
\hline 17 & Saxena & 2014 & $-308 \mathrm{G} / \mathrm{A}$ & Case control & 59 & 139 & India & PCR-RFLP & HBV & $\begin{array}{l}\text { Healthy } \\
\text { subjects }\end{array}$ & 8 \\
\hline 18 & Jin & 2015 & $\begin{array}{l}-1031 \mathrm{~T} / \mathrm{C},-857 \mathrm{C} / \mathrm{T} \\
-238 \mathrm{G} / \mathrm{A}\end{array}$ & Case control & 224 & 206 & Korea & $\begin{array}{l}\text { Single base } \\
\text { primer extension } \\
\text { assay }\end{array}$ & HBV & LC & 7 \\
\hline 19 & Sghaier & 2015 & $-308 \mathrm{G} / \mathrm{A},-238 \mathrm{G} / \mathrm{A}$ & Case control & 15 & 200 & Tunisia & PCR-RFLP & HBV & $\begin{array}{l}\text { Healthy } \\
\text { subjects }\end{array}$ & 8 \\
\hline 20 & Shin & 2015 & $\begin{array}{l}-1031 \mathrm{~T} / \mathrm{C},-863 \mathrm{C} / \mathrm{A} \\
-857 \mathrm{C} / \mathrm{T},-308 \mathrm{G} / \mathrm{A} \\
-238 \mathrm{G} / \mathrm{A}\end{array}$ & Case control & 157 & 201 & Korea & PCR-RFLP & Mixed & $\begin{array}{l}\text { Healthy } \\
\text { subjects }\end{array}$ & 8 \\
\hline 21 & Yang & 2015 & $\begin{array}{l}-1031 \mathrm{~T} / \mathrm{C},-863 \mathrm{C} / \mathrm{A} \\
-857 \mathrm{C} / \mathrm{T},-308 \mathrm{G} / \mathrm{A}\end{array}$ & Case control & 298 & 889 & Taiwan & PCR-RFLP & $\begin{array}{l}\text { Smoking } \\
\text { and } \\
\text { alcohol }\end{array}$ & $\begin{array}{l}\text { Healthy } \\
\text { subjects }\end{array}$ & 7 \\
\hline 22 & Tsai & 2017 & $-308 \mathrm{G} / \mathrm{A}$ & Case control & 200 & 200 & Taiwan & Sequencing & HBV & LC & 6 \\
\hline 23 & Tharwat & 2018 & $-308 \mathrm{G} / \mathrm{A}$ & $\begin{array}{l}\text { Retrospective } \\
\text { cohort }\end{array}$ & 29 & 16 & Egypt & PCR-RFLP & $\mathrm{HCV}$ & $\begin{array}{l}\text { Healthy } \\
\text { subjecys }\end{array}$ & 8 \\
\hline
\end{tabular}


Table 2 Allele frequencies of SNPs in each population

\begin{tabular}{|c|c|c|c|c|c|c|c|c|c|c|}
\hline \multirow[t]{2}{*}{ SNP } & \multicolumn{2}{|c|}{ Eastern Asia } & \multicolumn{2}{|c|}{ Southern Asia } & \multicolumn{2}{|l|}{ Africa } & \multicolumn{2}{|l|}{ Europe } & \multicolumn{2}{|c|}{ South America } \\
\hline & $\mathrm{HCC}$ & Control & $\mathrm{HCC}$ & Control & $\mathrm{HCC}$ & Control & $\mathrm{HCC}$ & Control & $\mathrm{HCC}$ & Control \\
\hline$-1031 C$ & $22.12 \%$ & $21.75 \%$ & - & - & - & - & - & - & - & - \\
\hline$-863 \mathrm{~A}$ & $15.67 \%$ & $14.62 \%$ & $39 \%$ & $32.98 \%$ & - & - & - & - & - & - \\
\hline$-857 \mathrm{~T}$ & $19.25 \%$ & $18.21 \%$ & $14.29 \%$ & $12.94 \%$ & - & - & - & - & - & - \\
\hline$-308 \mathrm{~A}$ & $13.68 \%$ & $8.73 \%$ & $6.88 \%$ & $6.75 \%$ & $25.58 \%$ & $26.33 \%$ & $18.64 \%$ & $5 \%$ & $13.51 \%$ & $7.92 \%$ \\
\hline$-238 \mathrm{~A}$ & $5.97 \%$ & $4.64 \%$ & $9.38 \%$ & $8.51 \%$ & $66.67 \%$ & $45.75 \%$ & - & - & $18.47 \%$ & $9.65 \%$ \\
\hline
\end{tabular}

\section{Association between SNP TNF-a - 238 and HCC risk}

Nine studies with 831 cases and 1293 controls were included to determine the association between SNP TNF$\alpha-238$ and HCC risk. Similar to the TNF-a - 863 SNP, the numbers of cases for GA and AA genotypes were reported together in the study by Niro et al. [42], thus it could only be used for dominant-model analysis (GA/ AA vs GG; Table 3). In studies with heterogeneity among studies $\left(\mathrm{I}_{2}\right)$ more than $60 \%$ and $p$-value for the heterogeneity was less than 0.05 , we applied random-

Table 3 Pooled risk estimates for SNPs TNF-a -1031 T/C, -863 C/A, -857 C/T, -308 G/A, and - 238 G/A with HCC risk

\begin{tabular}{|c|c|c|c|c|c|}
\hline SNP TNF-a & $\mathrm{n}$ & OR $(95 \% \mathrm{Cl})$ & $p$ value for $Z$ test & $I^{2}$ for heterogenity & $p$ value for heterogenity \\
\hline \multicolumn{6}{|l|}{$-1031 \mathrm{~T} / \mathrm{C}$} \\
\hline C vs T & 3 & 1.06 [0.89-1.26] & 0.52 & 0.77 & 0 \\
\hline $\mathrm{CC}+\mathrm{TC}$ vs TT & 5 & $1.04[0.86-1.27]$ & 0.69 & 0.97 & 0 \\
\hline CC vs TT & 3 & 1.39 [0.88-2.18] & 0.15 & 0.99 & 0 \\
\hline$C C$ vs TC $+\pi$ & 3 & $1.41[0.91-2.21]$ & 0.13 & 1 & 0 \\
\hline TC vs TT & 3 & $0.96[0.77-1.19]$ & 0.70 & 0.64 & 0 \\
\hline \multicolumn{6}{|l|}{$-863 \mathrm{C} / \mathrm{A}$} \\
\hline A vs $C$ & 7 & 1.31 [1.03-1.67] & $0.03^{*}$ & 0.007 & 66 \\
\hline$A A+C A$ vs $C C$ & 8 & 1.19 [1.03-1.36] & $0.02^{*}$ & 0.35 & 10 \\
\hline AA vs CC & 7 & $1.43[0.98-2.10]$ & 0.07 & 0.50 & 0 \\
\hline$A A$ vs $C A+C C$ & 7 & 1.28 [0.89-1.86] & 0.19 & 0.84 & 0 \\
\hline CA vs CC & 7 & 1.41 [1.00-1.99] & 0.05 & 0.0007 & 74 \\
\hline \multicolumn{6}{|l|}{$-857 \mathrm{C} / \mathrm{T}$} \\
\hline T vs $C$ & 4 & 1.16 [0.94-1.43] & 0.17 & 0.45 & 0 \\
\hline$\pi+C T$ vs $C C$ & 5 & 1.31 [1.06-1.62] & $0.01^{*}$ & 0.24 & 27 \\
\hline$\pi$ vs CC & 4 & $0.75[0.42-1.36]$ & 0.35 & 0.87 & 0 \\
\hline$\Pi$ vs $C T+C C$ & 4 & $0.77[0.44-1.35]$ & 0.36 & 0.77 & 0 \\
\hline CT vs CC & 4 & $1.02[0.77-1.34]$ & 0.89 & 0.54 & 0 \\
\hline \multicolumn{6}{|l|}{$-308 \mathrm{G} / \mathrm{A}$} \\
\hline$A$ vs $G$ & 17 & 1.98 [1.62-2.42] & $<0.001^{*}$ & 0.03 & 44 \\
\hline $\mathrm{AA}+\mathrm{GA}$ vs $\mathrm{GG}$ & 19 & 1.95 [1.53-2.49] & $<0.001^{*}$ & 0.003 & 54 \\
\hline AA vs GG & 13 & 3.14 [2.06-4.79] & $<0.001^{*}$ & 0.69 & 0 \\
\hline$A A$ vs $G A+G G$ & 13 & $2.52[1.69-3.76]$ & $<0.001^{*}$ & 0.81 & 0 \\
\hline GA vs GG & 17 & $2.07[1.60-2.68]$ & $<0.001^{*}$ & 0.006 & 52 \\
\hline \multicolumn{6}{|l|}{$-238 \mathrm{G} / \mathrm{A}$} \\
\hline$A$ vs $G$ & 8 & 1.50 [1.16-1.94] & $0.002^{*}$ & 0.07 & 46 \\
\hline$A A+G A$ vs $G G$ & 9 & $1.39[0.87-2.24]$ & 0.17 & 0.03 & 52 \\
\hline AA vs GG & 5 & 3.87 [1.32-11.34] & $0.01^{*}$ & 0.70 & 0 \\
\hline$A A$ vs $G A+G G$ & 8 & $2.67[1.17-6.10]$ & $0.02^{*}$ & 0.87 & 0 \\
\hline GA vs GG & 8 & 1.28 [0.72-2.28] & 0.39 & 0.01 & 61 \\
\hline
\end{tabular}




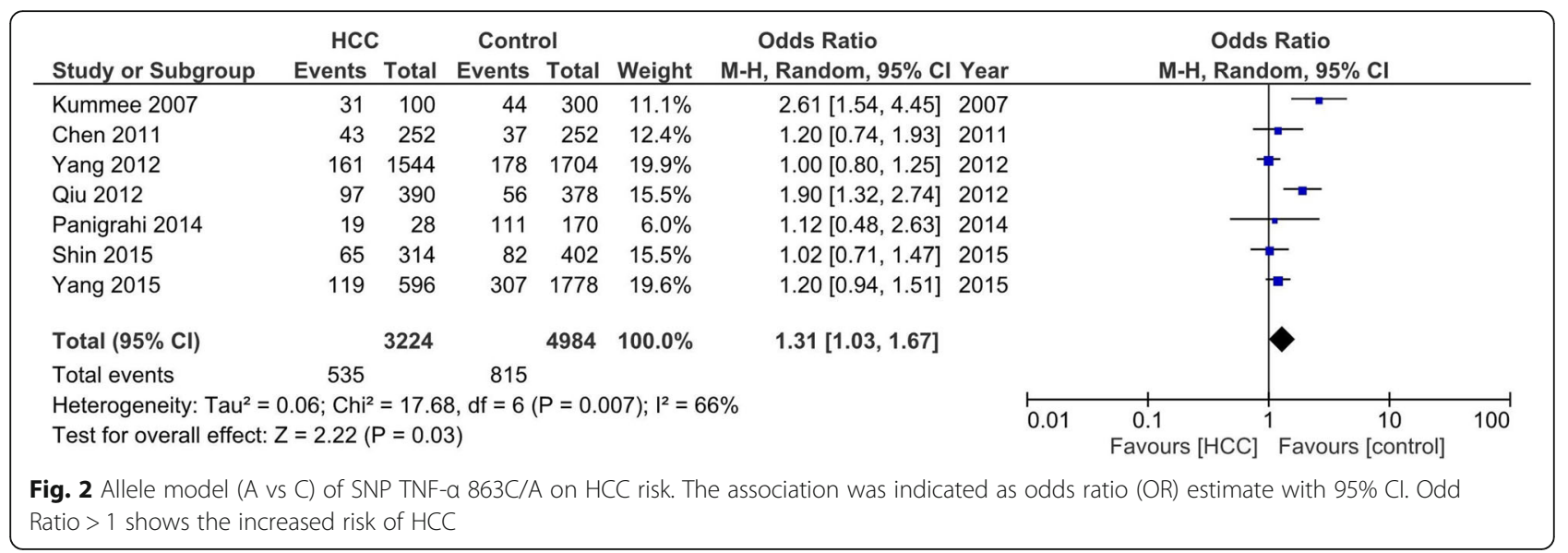

effects models. The allele model of A versus G of SNP TNF- $\alpha-238 \mathrm{G} / \mathrm{A}$ was significantly associated with risk of $\mathrm{HCC}(\mathrm{OR}=1.50,95 \% \mathrm{CI}=1.16-1.94, p=0.002)$. The codominant-model analysis (AA vs GG) also showed significant association between SNP TNF- $\alpha 238 \mathrm{G} / \mathrm{A}$ and $\mathrm{HCC}$ risk $(\mathrm{OR}=3.87,95 \% \mathrm{CI}=1.32-11.34, p=0.01)$. The recessive model analysis (AA vs $\mathrm{GA}+\mathrm{GG}$ ) proved significant association between SNP TNF- $\alpha 238 \mathrm{G} / \mathrm{A}$ and HCC risk as well $(\mathrm{OR}=2.67,95 \% \mathrm{CI}=1.17-6.10, p=$ 0.02; Fig. 5). As heterogeneity was found in the statistical analyses, we did sensitivity analyses to evaluate the sources of heterogeneity. We found that heterogeneity between studies was mainly caused by the study by Teixeira et al. [41], as after this study was excluded, no significant heterogeneity was found.

\section{Sensitivity analysis}

In this meta-analysis, there were no significant change in ORs by deleting a particular study, which indicated that no single study influenced the statistical significance of the overall results.

\section{Discussion}

The development of HCC depends on some factors such as viral infection, environmental, behavioral (smoking, alcoholism), metabolism, and genetics $[44,45]$. The contribution of SNP as a genetic factor is widely studied related to its role in the development of HCC, in which the prevalence of SNP is different in each population [5, $46,47]$. From the host factor, TNF- $\alpha$ is thought to play an important role in hepatocarcinogenesis through necroinflammation and the induction of fibrogenic factors [48]. Five biallelic SNPs in the promoter region Tumor Necrosis Factor- $\alpha$ gene were known: $-1031 \mathrm{~T} / \mathrm{C}$, $-863 \mathrm{C} / \mathrm{A},-857 \mathrm{C} / \mathrm{T},-308 \mathrm{G} / \mathrm{A}$, and $-238 \mathrm{G} / \mathrm{A}$ [7]. However, to date, only few meta-analyses have analysed all these five SNPs with HCC risk. Hereby, we performed this updated meta-analysis to clarify the independent role of each of these five SNPs on HCC risk.

As research on SNP TNF- $\alpha-1031 \mathrm{~T} / \mathrm{C}$ and HCC risk is still limited, we only found five studies investigating this SNP, even though many studies have shown significant association between this SNP with other diseases such as polycystic ovary syndrome and endometriosis $[49,50]$. In a study conducted by Shin et al. survival of HCC cases with TNF- $\alpha-1031$ wild type (TT) genotype or SNP TC genotype was significantly better than those with the SNP CC genotype [8]. However, in this metaanalysis, no significant relationship was found between the SNP and HCC risk in all genetic models. Meta-

\begin{tabular}{|c|c|c|c|c|c|c|c|c|c|c|c|}
\hline Study or Subgroup & \multicolumn{2}{|c|}{$\mathrm{HCC}$} & \multicolumn{2}{|c|}{ Control } & \multicolumn{2}{|r|}{ Odds Ratio } & \multicolumn{5}{|c|}{$\begin{array}{c}\text { Odds Ratio } \\
\text { M-H, Fixed, } 95 \% \mathrm{Cl}\end{array}$} \\
\hline Chen 2011 & 31 & 126 & 28 & 126 & $13.9 \%$ & $1.14[0.64,2.05]$ & 2011 & & 7 & $=$ & \\
\hline giu 2012 & 90 & 195 & 136 & 376 & $32.8 \%$ & $1.51[1.06,2.15]$ & 2012 & & & $=$ & \\
\hline Panigrahi 2014 & 4 & 14 & 27 & 96 & $3.2 \%$ & $1.02[0.30,3.54]$ & 2014 & & & & \\
\hline Shin 2015 & 41 & 157 & 59 & 201 & $25.1 \%$ & $0.85[0.53,1.36]$ & 2015 & & $\rightarrow$ & t & \\
\hline $\operatorname{Jin} 2015$ & 94 & 224 & 63 & 206 & $25.0 \%$ & $1.64[1.10,2.44]$ & 2015 & & & $\rightarrow-$ & \\
\hline Total $(95 \% \mathrm{Cl})$ & & 716 & & 1005 & $100.0 \%$ & $1.31[1.06,1.62]$ & & & & $\bullet$ & \\
\hline Total events & 260 & & 313 & & & & & & & & \\
\hline $\begin{array}{l}\text { Heterogeneity: } \mathrm{Chi}^{2}= \\
\text { Test for overall effect }\end{array}$ & $\begin{array}{l}5.51, \mathrm{df}= \\
Z=2.54\end{array}$ & $\begin{array}{l}4(\mathrm{P}= \\
P=0.0\end{array}$ & $\begin{array}{l}0.24) ; i^{2}= \\
\text { 11) }\end{array}$ & $27 \%$ & & & & $\lcm{0.01}$ & $\begin{array}{c}1 \\
0.1 \\
\text { Favours }[\mathrm{HCC}]\end{array}$ & $\begin{array}{c}10 \\
10 \\
\text { Favours [control] }\end{array}$ & 100 \\
\hline
\end{tabular}




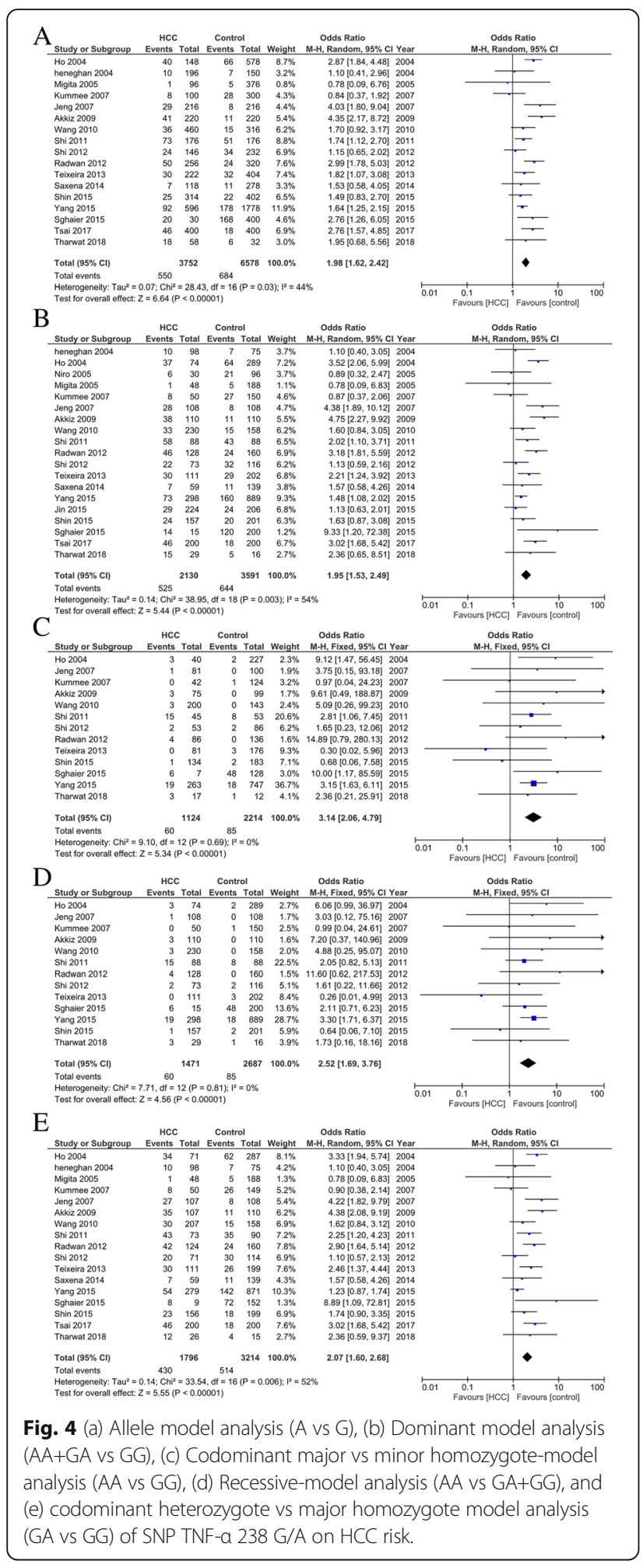

analysis conducted by Wei et al. also shows that there is no significant relationship between this SNP and HCC risk [51].

This study shows a significant relationship between SNP TNF- $\alpha-863 \mathrm{C} / \mathrm{A}$ with HCC risk in allele models and dominant model analysis. A meta-analysis conducted by
Wei et al. also showed a significant relationship betweehis SNP and HCC in dominant and codominant (CA vs CC) model analysis [51]. Polymorphism of TNF- $\alpha-863 \mathrm{C} / \mathrm{A}$ in the promoter region can influence TNF- $\alpha$ expression, however, the result is still conflicting. Some research suggest that it may increase TNF- $\alpha$ expression [52, 53], while other research show the opposite result in which it may decrease TNF- $\alpha$ expression $[30,54]$. A study by Skoog et al. proposed that TNF- $\alpha-863$ C/A polymorphism affects the binding of nuclear protein(s) to the promoter region of the TNF- $\alpha$ gene, with accompanying changes in TNF- $\alpha$ expression, thus leading to variation in TNF- $\alpha$ levels [55]. The role of ethnicity may also play a role as well, carriers of the rare ' $A$ ' allele have a significantly lower TNF- $\alpha$ levels in Swedish and Indian population [55, 56], while they have a significantly higher levels in Japanese population $[52,53]$.

In the present study, we also found a significant relationship between SNP TNF- $\alpha-857 \mathrm{C} / \mathrm{T}$ with dominant model analysis. Limited studies were present regarding the relationship between this polymorphism with HCC risk, thus there were only 7 studies that came within our inclusion criteria. This is different from the metaanalysis conducted by Wei et al. which showed no significant relationship between this SNP and HCC risk, however in that meta-analysis there were only 3 included studies [51].

There have been many previous studies investigating SNP TNF- $\alpha-308$ G/A with HCC risk, thus we got 19 included studies. All five genetic analysis models showed a significant relationship between the SNP and HCC risk. This is in line with several previous meta-analysis studies, including those conducted by $\mathrm{Hu}$ et al. and Tavakolpour and Sali on allele models and dominant model analyses [17, 57]. Wei et al. on codominant and dominant model analyses [51], and Xiao et al. in all except recessive model analysis [18]. Of all other TNF- $\alpha$ SNPs, -308 G/A is the most studied SNP. This SNP is also correlated with the risk of other cancer, such as breast cancer and gastric cancer [56, 58]. This SNP seems to be variable within ethnicities, as in metaanalysis study conducted by Wei et al., SNP - 308 AA was associated with an increased risk of $\mathrm{HCC}$ in Asian ethnicities, but not for Caucasian [51]. A study conducted by Shin et al. on South Korean population showed that TNF- $\alpha-308$ SNP alone was not significantly associated with HCC, but when several genotypes were combined (e.g. -1031 / - 308 / -238 TT / GG / $\mathrm{GA})$, there was a significant association with the incidence of HCC [8].

Studies on SNP TNF- $\alpha-238$ G/A have also been extensively performed. Nevertheless, meta-analyses of these SNPs are still limited and yield conflicting results. The present study showed a significant relationship 


\begin{tabular}{|c|c|c|c|c|c|c|c|c|c|c|}
\hline \multirow{2}{*}{$\begin{array}{l}\text { Study or Subgroup } \\
\text { Kummee } 2007\end{array}$} & \multirow{2}{*}{$\begin{array}{r}\begin{array}{r}\mathrm{HCC} \\
\text { Events }\end{array} \\
1\end{array}$} & \multirow{2}{*}{$\begin{array}{r}\text { Total } \\
45\end{array}$} & \multirow{2}{*}{$\begin{array}{r}\begin{array}{r}\text { Contr } \\
\text { Events }\end{array} \\
0\end{array}$} & \multirow{2}{*}{$\begin{array}{l}\text { Total } \\
140\end{array}$} & \multirow{2}{*}{$\begin{array}{r}\text { Weight } \\
6.6 \%\end{array}$} & $\begin{array}{l}\text { Odds Ratio } \\
\text { M-H, Fixed, } 95 \% \text { Cl Year }\end{array}$ & \multicolumn{4}{|c|}{$\begin{array}{c}\text { Odds Ratio } \\
\text { M-H, Fixed, } 95 \% \mathrm{Cl}\end{array}$} \\
\hline & & & & & & $9.47[0.38,236.67] 2007$ & & & 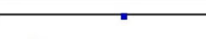 & $\rightarrow$ \\
\hline Wang 2010 & 1 & 210 & 0 & 139 & $16.5 \%$ & $2.00[0.08,49.39] 2010$ & & & & \\
\hline Panigrahi 2014 & 2 & 13 & 3 & 61 & $24.7 \%$ & $3.52[0.52,23.54] 2014$ & & & & \\
\hline Shin 2015 & 1 & 131 & 1 & 105 & $30.5 \%$ & $0.80[0.05,12.94] 2015$ & & $=$ & & \\
\hline Sghaier 2015 & 6 & 7 & 45 & 107 & $21.8 \%$ & $8.27[0.96,71.08] 2015$ & & & & \\
\hline Total $(95 \% \mathrm{Cl})$ & & 406 & & 552 & $100.0 \%$ & $3.87[1.32,11.34]$ & & & & \\
\hline Total events & 11 & & 49 & & & & & & & \\
\hline $\begin{array}{l}\text { Heterogeneity: } \mathrm{Ch}^{2}= \\
\text { Test for overall effect: }\end{array}$ & $\begin{array}{l}.18, \mathrm{df}= \\
=2.46(F\end{array}$ & $\begin{array}{l}4(P=C \\
=0.0\end{array}$ & 0.70); $1^{2}=$ & $0 \%$ & & & 0.01 & $\begin{array}{c}0.1 \\
\text { Favours }[\mathrm{HCC}]\end{array}$ & $\begin{array}{c}10 \\
\text { Favours [control] }\end{array}$ & $\overrightarrow{100}$ \\
\hline
\end{tabular}

between this SNP and HCC risk. This is in line with a meta-analysis conducted by Xiao et al. showing that this SNP is associated with HCC risk, although in that study only HBV-related HCC was studied [18]. Another metaanalysis conducted by $\mathrm{Hu}$ et al., however, shows no significant relationship between this SNP with HCC risk in Asian population [57].

Those SNPs on TNF- $\alpha$ promoter can influence TNF- $\alpha$ level. They resulted in higher and constitutive TNF- $\alpha$ expression and were associated with an increased risk of HCC $[10,17]$. TNF- $\alpha$ itself is a potent pro-inflammatory cytokine [17, 59]. Necroinflammation in hepatocytes triggers mutagenesis and activation of oncogenes from proto-oncogenes in host cells, causing HCC [48]. In addition, through the chronic inflammatory pathway, TNF- $\alpha$ is also known to induce HCC through activation and differentiation of hepatic progenitor cells [60].

This meta-analysis still had several limitations. To date, there has rarely been any meta-analysis discussing the relationship between all each SNPs of TNF- $\alpha-1031$ T/C, $-863 \mathrm{C} / \mathrm{A},-857 \mathrm{C} / \mathrm{T}$, and $-238 \mathrm{G} / \mathrm{A}$ with $\mathrm{HCC}$. The second limitation of this study was that we only included studies in English language so that it does not rule out the exclusion of any good research in nonEnglish languages. Third, the role of other factors, such as variations in genes adjacent to the TNF- $\alpha$ gene or epigenetic factors are said to also be able to regulate TNF- $\alpha$ expression [61, 62]. Fourth, our study is a meta-analysis so it is not possible to generate a per-patient haplotype analysis. Further research is required to determine the effects of these various TNF- $\alpha$ SNPs in each different population.

\section{Conclusion}

In conclusion, this meta-analysis determined the association between SNP TNF- $\alpha-1031$ T/C, -863 C/A, -857 $\mathrm{C} / \mathrm{T},-308 \mathrm{G} / \mathrm{A}$, and $-238 \mathrm{G} / \mathrm{A}$ and HCC risk. Our novel data demonstrated that all these SNPs but $-1031 \mathrm{~T} / \mathrm{C}$ might increase HCC risk. Therefore, genetic predisposition, especially polymorphism of TNF- $\alpha$ gene may play important role in the pathogenesis of HCC. Further studies in larger population and analysing geneenvironment interaction are required to provide better understanding between TNF- $\alpha$ polymorphisms and the risk of $\mathrm{HCC}$.

\section{Supplementary Information}

The online version contains supplementary material available at https://doi. org/10.1186/s12885-020-07606-6.

\section{Additional file 1.}

\section{Abbreviations}

HCC: Hepatocellular carcinoma; TNF-a: Tumor Necrosis Factor-a; SNP: Single Nucleotide Polymorphism; HBV: Hepatitis B Virus; HCV: Hepatitis C Virus; RR: Relative risk; OR: Odds ratio; Cl: Confidence interval; NOQS: Newcastle Ottawa quality assessment scale

\section{Acknowledgments \\ Not applicable.}

\section{Authors' contributions}

This study was designed by CDKW and $\mathrm{RH}, \mathrm{CDKW}$ and FCA conducted the literature search, S performed statistical analysis, GIP and RH wrote the manuscript. All authors read and approved the final manuscript.

\section{Funding}

All publication funds from the beginning of data collection to manuscript preparation and our publication costs are supported by Dato 'Sri Prof. Dr. Tahir through the Tahir Professorship Program, Indonesia.

\section{Availability of data and materials}

The datasets used and/or analyzed during the current study are available from the corresponding author on reasonable request.

\section{Ethics approval and consent to participate}

Not applicable.

Consent for publication

Not applicable.

\section{Competing interests}

The authors declare that they have no conflict of interest.

\section{Author details}

'Department of Medical Biochemistry, Faculty of Medicine, Universitas Airlangga, Surabaya, Indonesia. Institute of Tropical Disease, Universitas Airlangga, Surabaya, Indonesia. ${ }^{3}$ Faculty of Nursing, Universitas Jember, Jember, Indonesia. ${ }^{4}$ Hearing Vision Ltd-Darmo General Hospital, Surabaya, Indonesia. 
Received: 1 May 2020 Accepted: 31 October 2020

Published online: 23 November 2020

\section{References}

1. Bray F, Ferlay J, Soerjomataram I, Siegel RL, Torre LA, Jemal A. Global cancer statistics 2018: GLOBOCAN estimates of incidence and mortality worldwide for 36 cancers in 185 countries. CA Cancer J Clin. 2018;68(6):394-424. https://doi.org/10.3322/caac.21492.

2. WHO (2016) Cancer fact sheets: Liver cancer. http://gco.iarc.fr/today/data/ pdf/fact-sheets/cancers/cancer-fact-sheets-7.pdf. Accessed 1 Apr 2020

3. Brar TS, Hilgenfeldt E, Soldevila-Pico C. Etiology and pathogenesis of hepatocellular carcinoma. In: Liu C, editor. Precision molecular pathology of liver Cancer. Cham: Springer International Publishing; 2018. p. 1-15. https:// doi.org/10.1007/978-3-319-68082-8_1.

4. Nahon P, Zucman-Rossi J. Single nucleotide polymorphisms and risk of hepatocellular carcinoma in cirrhosis. J Hepatol. 2012;57(3):663-74. https:// doi.org/10.1016/j.jhep.2012.02.035.

5. Mathew S, Abdel-Hafiz H, Raza A, Fatima K, Qadri I. Host nucleotide polymorphism in hepatitis B virus-associated hepatocellular carcinoma. World J Hepatol. 2016;8(10):485-98. https://doi.org/10.4254/wjh.v8.i10. 485.

6. Ma L, Chen S, Mao X, Lu Y, Zhang X, Lao X, Qin X, Li S. The association between TNFR gene polymorphisms and the risk of hepatitis $B$ virus-related liver diseases in Chinese population. Sci Rep. 2018;8(1):9240. https://doi.org/ 10.1038/s41598-018-27623-7.

7. Chen X, Zhang L, Chang Y, Shen T, Wang L, Zhuang H, Lu F. Association of TNF-alpha genetic polymorphisms with hepatocellular carcinoma susceptibility: a case-control study in a Han Chinese population. Int J Biol Markers. 2011;26(3):181-7. https://doi.org/10.5301/jbm.2011.8580.

8. Shin SP, Kim NK, Kim JH, Lee JH, Kim JO, Cho SH, Park H, Kim MN, Rim KS, Hwang SG. Association between hepatocellular carcinoma and tumor necrosis factor alpha polymorphisms in South Korea. World J Gastroenterol. 2015;21(46):13064-72. https://doi.org/10.3748/wjg.v21.i46.13064.

9. El-Tahan RR, Ghoneim AM, El-Mashad N. TNF-a gene polymorphisms and expression. SpringerPlus. 2016;5(1):1508. https://doi.org/10.1186/s40064-0163197-y.

10. Cheong JY, Cho SW, Hwang IL, Yoon SK, Lee JH, Park CS, Lee JE, Hahm KB, Kim JH. Association between chronic hepatitis B virus infection and interleukin-10, tumor necrosis factor-a gene promoter polymorphisms. J Gastroenterol Hepatol. 2006;21(7):1163-9. https://doi.org/10.1111/j.14401746.2006.04304.x.

11. Koziel MJ, Dudley D, Afdhal N, Grakoui A, Rice CM, Choo QL, Houghton M, Walker BD. HLA class I-restricted cytotoxic T lymphocytes specific for hepatitis $C$ virus. Identification of multiple epitopes and characterization of patterns of cytokine release. J Clin Invest. 1995;96(5):2311-21. https://doi. org/10.1172/jci118287.

12. Martins GR, Gelaleti GB, Moschetta MG, Maschio-Signorini LB, Zuccari DA. Proinflammatory and anti-inflammatory cytokines mediated by NF-KB factor as prognostic markers in mammary tumors. Mediat Inflamm. 2016;2016: 9512743. https://doi.org/10.1155/2016/9512743.

13. Méndez-García LA, Nava-Castro KE, Ochoa-Mercado TL, Palacios-Arreola MI, Ruiz-Manzano RA, Segovia-Mendoza M, Solleiro-Villavicencio H, CázarezMartínez C, Morales-Montor J. Breast Cancer metastasis: are cytokines important players during its development and progression? J Interferon Cytokine Res. 2019;39(1):39-55. https://doi.org/10.1089/jir.2018.0024.

14. Wang Z, Li Z, Ye Y, Xie L, Li W. Oxidative stress and liver Cancer: etiology and therapeutic targets. Oxidative Med Cell Longev. 2016;2016:7891574. https://doi.org/10.1155/2016/7891574.

15. Wang H, Liu J, Hu X, Liu S, He B. Prognostic and therapeutic values of tumor necrosis factor-alpha in hepatocellular carcinoma. Med Sci Monitor. 2016;22:3694-704. https://doi.org/10.12659/msm.899773.

16. Jeng J-E, Tsai J-F, Chuang L-Y, Ho M-S, Lin Z-Y, Hsieh M-Y, Chen S-C, Chuang $W-L$, Wang $L-Y, Y u$ M-L, Dai $C-Y$, Chang J-G. Tumor necrosis factoralpha 308.2 polymorphism is associated with advanced hepatic fibrosis and higher risk for hepatocellular carcinoma. Neoplasia. 2007;9(11):987-92. https://doi.org/10.1593/neo.07781.

17. Tavakolpour S, Sali S. Tumor necrosis factor-a-308 G/a polymorphisms and risk of hepatocellular carcinoma: a meta-analysis. Hepat Mon. 2016;16(4): e33537. https://doi.org/10.5812/hepatmon.33537.

18. Xiao Q, Fu B, Chen P, Liu ZZ, Wang W, Ye Q. Three polymorphisms of tumor necrosis factor-alpha and hepatitis B virus related hepatocellular carcinoma: a meta-analysis. Medicine (Baltimore). 2016;95(50):e5609. https://doi.org/10. 1097/MD.0000000000005609.

19. Saxena R, Chawla YK, Verma I, Kaur J. IFN- $\gamma(+874)$ and not TNF-a (-308) is associated with HBV-HCC risk in India. Mol Cell Biochem. 2014;385(1):297307. https://doi.org/10.1007/s11010-013-1838-9.

20. Somi MH, Najafi L, Noori BN, Alizadeh AH, Aghah MR, Shavakhi A, Ehsani MJ, Aghazadeh R, Masoodi M, Amini S, Baladast M, Zali MR. Tumor necrosis factor-alpha gene promoter polymorphism in Iranian patients with chronic hepatitis B. Indian J Gastroenterol. 2006;25(1):14-5.

21. Heidari Z, Moudi B, Mahmoudzadeh Sagheb H, Moudi M. Association of TNF-a gene polymorphisms with production of protein and susceptibility to chronic hepatitis B infection in the south east Iranian population. Hepat Mon. 2016;16(11):e41984. https://doi.org/10.5812/hepatmon.41984.

22. Talaat RM, Esmail AA, Elwakil R, Gurgis AA, Nasr MI. Tumor necrosis factoralpha -308G/a polymorphism and risk of hepatocellular carcinoma in hepatitis C virus-infected patients. Chinese J Cancer. 2012;31(1):29-35. https://doi.org/10.5732/cjc.011.10258

23. Moher D, Liberati A, Tetzlaff J, Altman DG. Preferred reporting items for systematic reviews and meta-analyses: the PRISMA statement. PLoS Med. 2009;6(7):e1000097. https://doi.org/10.1371/journal.pmed.1000097.

24. Wells GA, Shea B, O'connell D, Peterson J, Welch V, Losos M, Tugwell P. The Newcastle-Ottawa scale (NOS) for assessing the quality of nonrandomised studies in meta-analyses 2013; 2016.

25. Heneghan MA, Johnson PJ, Clare M, Ho S, Harrison PM, Donaldson PT Frequency and nature of cytokine gene polymorphisms in hepatocellular carcinoma in Hong Kong Chinese. Int J Gastrointestinal Cancer. 2003;34(1): 19-26. https://doi.org/10.1385/IJGC:34:1:19.

26. Wang B, Wang J, Zheng Y, Zhou S, Zheng J, Wang F, Ma X, Zeng Z. A study of TNF-alpha-238 and -308 polymorphisms with different outcomes of persistent hepatitis B virus infection in China. Pathology. 2010;42(7):674-80. https://doi.org/10.3109/00313025.2010.523696

27. Shi HZ, Ren P, Lu QJ, Niedrgethmnn M, Wu GY. Association between EGF, TGF- $\beta 1$ and TNF-a gene polymorphisms and hepatocellular carcinoma. Asian Pacific J Cancer Prev. 2012;13(12):6217-20. https://doi.org/10.7314/ apjcp.2012.13.12.6217.

28. Qiu B, Wang X, Zhang P, Shi C, Zhang J, Qiu W, Wang W, Li D. Association of TNF-a promoter polymorphisms with the outcome of persistent HBV infection in a northeast Chinese Han population. Acta Biochim Biophys Sin. 2012;44(8):712-8. https://doi.org/10.1093/abbs/gms046.

29. Shi Z, Du C. Tumor necrosis factor alpha $308 \mathrm{G} / \mathrm{a}$ polymorphism and hepatocellular carcinoma risk in a Chinese population. Genet Testing Mol Biomarkers. 2011;15(7-8):569-72. https://doi.org/10.1089/gtmb.2011.0008.

30. Yang Y, Qiu X-Q, Yu H-P, Zeng X-Y, Bei C-H. TNF-a -863 polymorphisms and the risk of hepatocellular carcinoma. Exp Ther Med. 2012;3(3):513-8. https://doi.org/10.3892/etm.2011.418.

31. Ho S-Y, Wang Y-J, Chen H-L, Chen C-H, Chang C-J, Wang P-J, Chen HHW, Guo H-R. Increased risk of developing hepatocellular carcinoma associated with carriage of the TNF2 allele of the -308 tumor necrosis factor-a promoter gene. Cancer Causes Control. 2004;15(7):657-63. https://doi.org/ 10.1023/B:CACO.0000036173.99930.75

32. Yang MD, Hsu CM, Chang WS, Yueh TC, Lai YL, Chuang CL, Wang SC, Jeng LB, Ji HX, Hsiao CL, Wu CN, Tsai CW, Chung JG, Bau DT. Tumor necrosis factor-a genotypes are associated with hepatocellular carcinoma risk in Taiwanese males, smokers and alcohol drinkers. Anticancer Res. 2015;35(10):5417-23.

33. Tsai J-F, Chen S-C, Lin Z-Y, Dai C-Y, Huang J-F, Yu M-L, Chuang W-L. Independent and additive interaction between polymorphisms of tumor necrosis factor $a-308$ and lymphotoxin a+252 on risk of hepatocellular carcinoma related to hepatitis B. Kaohsiung J Med Sci. 2017;33(9):453-7. https://doi.org/10.1016/j.kjms.2017.04.009.

34. Panigrahi R, Sarkar N, Biswas A, Pal A, Saha D, Singh SP, Panigrahi MK, Bandopadhyay M, Chakrabarti S, Chakravarty R. Association of TNF-a promoter polymorphism with HBV associated disease outcome among HBV infected patients from Orissa, southern part of East India. J Clin Exp Hepatol. 2014;4(3):202-8. https://doi.org/10.1016/j.jceh.2014.08.002

35. Jin YJ, Lee D, Chung YH, Kim JA, Kim SE, Lee YS, Shin ES, Ryu SH, Jang MK, Lee JE, Park NH. Tumor necrosis factor-alpha gene polymorphism associated with development of hepatitis B virus-associated hepatocellular carcinoma. J Clin Gastroenterol. 2015;49(8):e76-81. https://doi.org/10.1097/mcg. 0000000000000261

36. Migita K, Miyazoe S, Maeda Y, Daikoku M, Abiru S, Ueki T, Yano K, Nagaoka S, Matsumoto T, Nakao K, Hamasaki K, Yatsuhashi H, Ishibashi H, Eguchi K. 
Cytokine gene polymorphisms in Japanese patients with hepatitis B virus infection--association between TGF-beta1 polymorphisms and hepatocellular carcinoma. J Hepatol. 2005;42(4):505-10. https://doi.org/10. 1016/j.hep.2004.11.026.

37. Kummee P, Tangkijvanich P, Poovorawan Y, Hirankarn N. Association of HLA-DRB1*13 and TNF-alpha gene polymorphisms with clearance of chronic hepatitis B infection and risk of hepatocellular carcinoma in Thai population. J Viral Hepat. 2007;14(12):841-8. https://doi.org/10.1111/j.13652893.2007.00880.x.

38. Akkiz H, Bayram S, Bekar A, Ozdil B, Akgöllü E, Sümbül AT, Demiryürek $H$, Doran F. G-308A TNF-alpha polymorphism is associated with an increased risk of hepatocellular carcinoma in the Turkish population: case-control study. Cancer Epidemiol. 2009;33(3-4):261-4. https://doi.org/10.1016/j.canep. 2009.06.001.

39. Radwan Ml, Pasha HF, Mohamed RH, Hussien HI, El-Khshab MN. Influence of transforming growth factor- $\beta 1$ and tumor necrosis factor-a genes polymorphisms on the development of cirrhosis and hepatocellular carcinoma in chronic hepatitis C patients. Cytokine. 2012;60(1):271-6. https://doi.org/10.1016/j.cyto.2012.05.010.

40. Tharwat E, Gad GFM, Nazmy MH, Mohamed HI, Hamza N, Wahid A, Ibrahim ARN. Impact of IL-27p28 (rs153109) and TNF-a (rs1800629) genetic polymorphisms on the progression of HCV infection in Egyptian patients. Immunol Investig. 2019;48(3):255-67. https://doi.org/10.1080/08820139.2018. 1510958.

41. Teixeira AC, Mendes CT Jr, Marano LA, Deghaide NH, Secaf M, Elias J Jr, Muglia V, Donadi EA, Martinelli AL. Alleles and genotypes of polymorphisms of IL-18, TNF- $\alpha$ and IFN- $\gamma$ are associated with a higher risk and severity of hepatocellular carcinoma (HCC) in Brazil. Hum Immunol. 2013;74(8):1024-9. https://doi.org/10.1016/j.humimm.2013.04.029.

42. Niro GA, Fontana R, Gioffreda D, Valvano MR, Lacobellis A, Facciorusso D, Andriulli A. Tumor necrosis factor gene polymorphisms and clearance or progression of hepatitis B virus infection. Liver Int. 2005;25(6):1175-81. https://doi.org/10.1111/j.1478-3231.2005.01166.x.

43. Sghaier I, Zidi S, Mouelhi L, Dabbech R, Ghazouani E, Brochot E, Stayoussef $M$, Yacoubi-Loueslati B. The relationship between TNF alpha gene polymorphisms (-238/-308), TNF RII VNTR (p75) and outcomes of hepatitis B virus infection in Tunisian population. Gene. 2015;568(2):140-5. https://doi org/10.1016/.j.gene.2015.05.029.

44. Zamor PJ, de Lemos AS, Russo MW. Viral hepatitis and hepatocellular carcinoma: etiology and management. J Gastrointest Oncol. 2017;8(2):22942. https://doi.org/10.21037/jgo.2017.03.14

45. An $P, X u J, Y u Y$, Winkler CA. Host and viral genetic variation in HBV-related hepatocellular carcinoma. Front Genet. 2018;9:261. https://doi.org/10.3389/ fgene.2018.00261.

46. Pontoriero AC, Trinks J, Hulaniuk ML, Caputo M, Fortuny L, Pratx LB, Frías A, Torres O, Nuñez F, Gadano A, Argibay P, Corach D, Flichman D. Influence of ethnicity on the distribution of genetic polymorphisms associated with risk of chronic liver disease in south American populations. BMC Genet. 2015; 16(1):93. https://doi.org/10.1186/s12863-015-0255-3.

47. Thursz M, Yee L, Khakoo S. Understanding the host genetics of chronic hepatitis B and C. Semin Liver Dis. 2011;31(2):115-27. https://doi.org/10. 1055/s-0031-1276642.

48. Aroucha DCBL, do Carmo RF, Moura P, Silva JLA, Vasconcelos LRS, Cavalcanti MSM, Muniz MTC, Aroucha ML, Siqueira ERF, Cahú GGOM, Pereira LMMB, Coêlho MRCD. High tumor necrosis factor-a/interleukin-10 ratio is associated with hepatocellular carcinoma in patients with chronic hepatitis C. Cytokine. 2013;62(3):421-5. https://doi.org/10.1016/j.cyto.2013.03.024.

49. Abutorabi R, Baradaran A, Sadat Mostafavi F, Zarrin Y, Mardanian F. Evaluation of tumor necrosis factor alpha polymorphism frequencies in endometriosis. Int J Fertil Steril. 2015;9(3):329-37. https://doi.org/10.22074/ ijfs.2015.4548.

50. Yun J-H, Choi J-W, Lee K-J, Shin J-S, Baek K-H. The promoter -1031(T/C) polymorphism in tumor necrosis factor-alpha associated with polycystic ovary syndrome. Reprod Biol Endocrinol. 2011;9(1):131. https://doi.org/10. 1186/1477-7827-9-131.

51. Wei Y, Liu F, Li B, Chen X, Ma Y, Yan L, Wen T, Xu M, Wang W, Yang J. Polymorphisms of tumor necrosis factor-alpha and hepatocellular carcinoma risk: a HuGE systematic review and meta-analysis. Dig Dis Sci. 2011;56(8):2227-36. https://doi.org/10.1007/s10620-011-1617-y.

52. Higuchi T, Seki N, Kamizono S, Yamada A, Kimura A, Kato H, Itoh K. Polymorphism of the $5^{\prime}$-flanking region of the human tumor necrosis factor
(TNF)-a gene in Japanese. Tissue Antigens. 1998;51(6):605-12. https://doi. org/10.1111/j.1399-0039.1998.tb03002.x.

53. Soga Y, Nishimura F, Ohyama H, Maeda H, Takashiba S, Murayama Y. Tumor necrosis factor-alpha gene (TNF-a) $-1031 /-863,-857$ single-nucleotide polymorphisms (SNPs) are associated with severe adult periodontitis in Japanese. J Clin Periodontol. 2003;30(6):524-31. https://doi.org/10.1034/j. 1600-051X.2003.00287.x.

54. Laddha NC, Dwivedi M, Begum R. Increased tumor necrosis factor (TNF)-a and its promoter polymorphisms correlate with disease progression and higher susceptibility towards vitiligo. PLoS One. 2012;7(12):e52298. https:// doi.org/10.1371/journal.pone.0052298.

55. Skoog T, van't Hooft FM, Kallin B, Jovinge S, Boquist S, Nilsson J, Eriksson P, Hamsten A. A common functional polymorphism ( $C \rightarrow$ a substitution at position -863) in the promoter region of the tumour necrosis factor-a (TNFa) gene associated with reduced circulating levels of TNF-a. Human Mol Genet. 1999;8(8):1443-9. https://doi.org/10.1093/hmg/8.8.1443.

56. Ahmad MM, Parveen F, Akhter N, Siddiqui JA, Shukla NK, Husain SA. Genetic polymorphism in TNF-a-308 G/a and TNF- $\beta+252$ a/G, as prognostic biomarker in breast Cancer patients among Indian population. Asian Pacific J Cancer Prev. 2020;21(2):301-8. https://doi.org/10.31557/apjcp.2020.21.2.301

57. Hu Q, Lou G-G, Liu Y-C, Qian L, LV B-D. The tumor necrosis factor-a-308 and -238 polymorphisms and risk of hepatocellular carcinoma for Asian populations: a meta-analysis. Curr Ther Res. 2014;76:70-5. https://doi.org/10. 1016/j.curtheres.2014.04.001.

58. Zheng W, Zhang S, Zhang S, Min L, Wang Y, Xie J, Hou Y, Tian X, Cheng J, Liu K, Xu D, Yu X, Liu Z, Lv Y, Liang N, Zhang J, Liu F, Tian Y. The relationship between tumor necrosis factor-a polymorphisms and gastric cancer risk: An updated meta-analysis. Biomed Rep. 2017;7(2):133-42. https://doi.org/10.3892/br.2017.934.

59. Barbosa MLC, Fumian MM, Miranda ALP, Barreiro EJ, Lima LM. Therapeutic approaches for tumor necrosis factor inhibition \%J Brazilian. J Pharm Sci. 2011:47:427-46.

60. Jing Y, Sun K, Liu W, Sheng D, Zhao S, Gao L, Wei L. Tumor necrosis factor-a promotes hepatocellular carcinogenesis through the activation of hepatic progenitor cells. Cancer Lett. 2018;434:22-32. https://doi.org/10.1016/j.canlet. 2018.07.001

61. Vázquez-Huerta DI, Alvarez-Rodríquez BA, Topete-Reyes JF, Muñoz-Valle JF, Parra-Michel R, Fuentes-Ramírez F, Salazar-López MA, Valle Y, Reyes-Castillo Z, Cruz-González A, Brennan-Bourdon LM, Torres-Carrillo N. Tumor necrosis factor alpha $-238 \mathrm{G} / \mathrm{a}$ and $-308 \mathrm{G} / \mathrm{a}$ polymorphisms and soluble TNF-a levels in chronic kidney disease: correlation with clinical variables. Int J Clin Exp Med. 2014;7(8):2111-9.

62. Wang Q, Roy B, Turecki G, Shelton RC, Dwivedi Y. Role of complex epigenetic switching in tumor necrosis factor-a Upregulation in the prefrontal cortex of suicide subjects. Am J Psychiatry. 2018;175(3):262-74. https://doi.org/10.1176/appi.ajp.2017.16070759.

\section{Publisher's Note}

Springer Nature remains neutral with regard to jurisdictional claims in published maps and institutional affiliations.

\section{Ready to submit your research? Choose BMC and benefit from:}

- fast, convenient online submission

- thorough peer review by experienced researchers in your field

- rapid publication on acceptance

- support for research data, including large and complex data types

- gold Open Access which fosters wider collaboration and increased citations

- maximum visibility for your research: over $100 \mathrm{M}$ website views per year

At BMC, research is always in progress.

Learn more biomedcentral.com/submissions 